\title{
Joint European Society of Paediatric Radiology (ESPR) and International Society for Forensic Radiology and Imaging (ISFRI) guidelines: paediatric postmortem computed tomography imaging protocol
}

\author{
Susan C. Shelmerdine ${ }^{1,2}$ (1) C Chandra Y. Gerrard ${ }^{3} \cdot$ Padma Rao $^{4} \cdot$ Matthew Lynch $^{4}$ - Jeroen Kroll ${ }^{5}$ - Dan Martin ${ }^{6}$. \\ Elka Miller ${ }^{7}$. Laura Filograna ${ }^{8} \cdot$ Rosa Maria Martinez $^{9}$. Odey Ukpo ${ }^{10}$ - Barry Daly ${ }^{11}$. Hideki Hyodoh ${ }^{12}$. \\ Karl Johnson ${ }^{13}$. Andrew Watt ${ }^{14}$. Ajay Taranath ${ }^{15}$. Scott Brown ${ }^{15}$. David Perry ${ }^{16}$. Lene Warner Thorup Boel ${ }^{17}$. \\ Aleksandra Borowska-Solonynko ${ }^{18}$ - Rick van Rijn ${ }^{19}$ • Willemijn Klein ${ }^{20}$ • Elspeth Whitby ${ }^{21}$ • Owen J. Arthurs ${ }^{1,2}$
}

Received: 11 October 2018 / Revised: 29 October 2018 / Accepted: 20 December 2018 / Published online: 28 February 2019

(C) The Author(s) 2019

\begin{abstract}
Postmortem CT for investigating childhood deaths is increasingly utilised as a noninvasive adjunct or alternative to standard autopsy; however there are no standardised published imaging protocols. This article describes a standardised imaging protocol that has been developed based on current practices of international postmortem imaging practitioners and experts. This recommendation is expected to be useful for postmortem imaging centres wishing to update their existing practices and for those starting paediatric postmortem $\mathrm{CT}$ as a new service.
\end{abstract}

Keywords Children $\cdot$ Computed tomography $\cdot$ Consensus recommendations $\cdot$ Paediatric $\cdot$ Postmortem $\cdot$ Protocol

\section{Introduction}

Postmortem CT is a relatively fast, inexpensive and widely accessible modality, commonly used in adults as part of routine postmortem examination $[1,2]$. The adaptation of this technique to evaluate childhood deaths is clearly a natural progression of the technology [3-5]. Compared to conventional paediatric autopsy practices, postmortem CT is noninvasive examination and provides a three-dimensional (3-D) digital record of anatomical information that can be postprocessed in a variety of ways for clinical, research and teaching purposes. This is particularly useful where sanitised images are required for a jury in medicolegal proceedings, or to show family members when explaining the cause of death [6]. Although few publications relate to the diagnostic accuracy rates of paediatric postmortem $\mathrm{CT}$, those published report reasonable concordance rates with autopsy of between $57.1 \%$

Owen J. Arthurs

owen.arthurs@gosh.nhs.uk

Extended author information available on the last page of the article and $83.3 \%[4,5,7,8]$, particularly for musculoskeletal abnormalities such as fractures.

Despite these advantages, there is much debate regarding appropriate referral indications, access to scanners, and precise techniques in performing paediatric postmortem CT. In the United Kingdom, the Royal College of Pathologists acknowledges the role that postmortem CT offers for the workup of sudden unexpected death in infancy, yet most referrals are performed on a case-by-case basis [9]. In the Netherlands, postmortem CT has been offered for all paediatric deaths by law since 2010 [10, 11]. Neither of these guidelines specifies the imaging parameters by which the examination should be conducted. A recent survey by the European Society of Paediatric Radiology (ESPR) on paediatric postmortem imaging [12] revealed that of the $24 / 47$ (51\%) centres reported to currently perform paediatric postmortem CT, only about half of these $(14 / 24$, or $58 \%)$ had a standardised CT protocol, with others reporting that protocols are modified and tailored each time for different patients. Furthermore, published studies in the literature report significant variation in image acquisition and provide insufficient information for this to be used as a template for other centres (Table 1) [3-5, 7, 8, 10, 13]. 


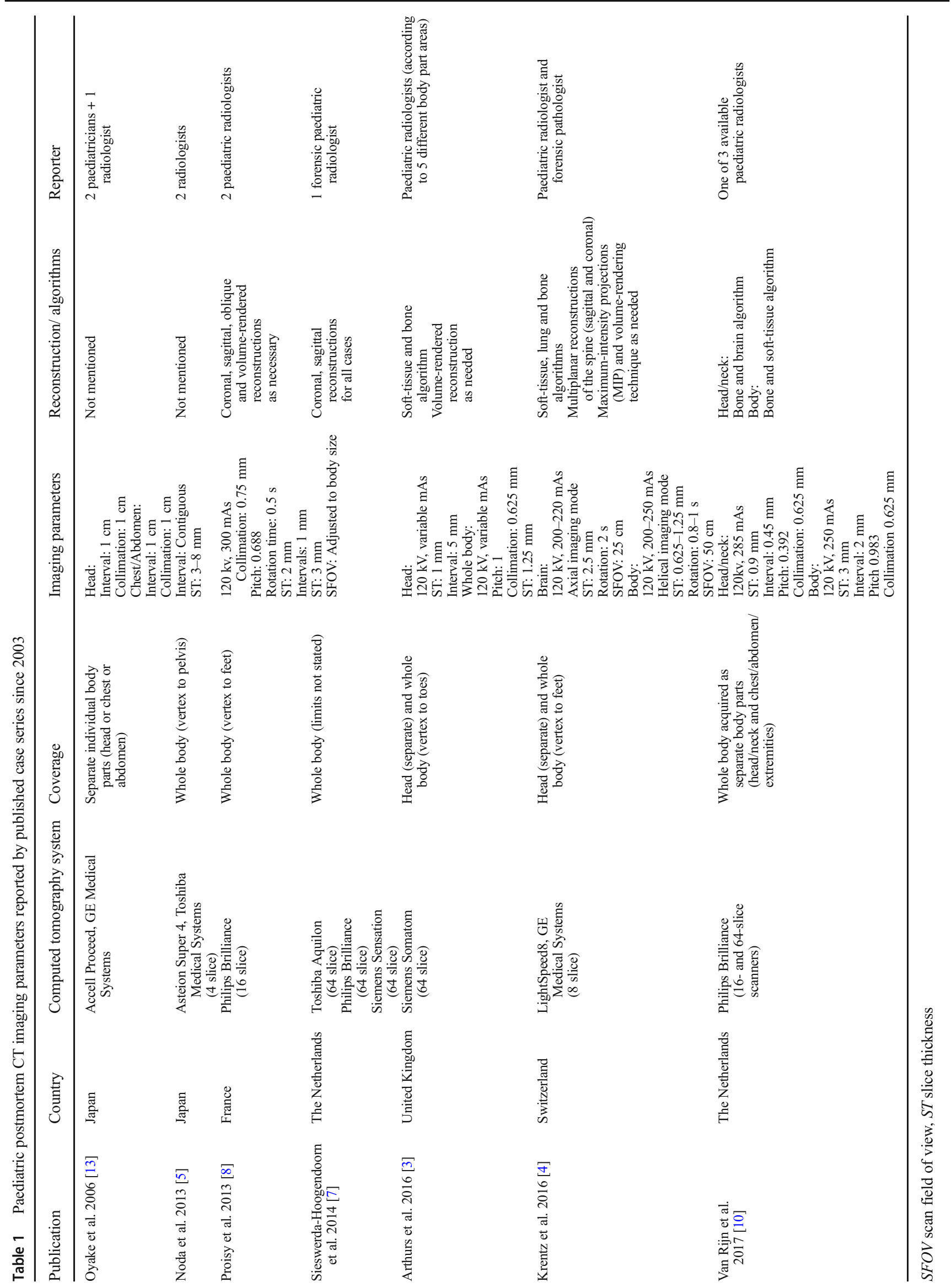


The aim of this study was therefore to develop a consensus imaging protocol for paediatric postmortem CT based on current international practices. This would enable standardisation of imaging acquisition, provide guidance for centres wishing to offer paediatric postmortem CT imaging, and aid uniformity of image quality for prospective multicentre review and research collaboration.

\section{Society surveys}

We designed a survey containing 20 questions relating to paediatric postmortem CT imaging referral patterns, imaging acquisition parameters, reconstruction algorithms and reporting practices. Potential responses included both multiple choice answers and free-text space to allow for detailed replies and elaboration on imaging techniques. We requested that responders also submit their own local departmental paediatric postmortem $\mathrm{CT}$ imaging protocols for review.

All registered members of the European Society of Paediatric Radiology (ESPR) postmortem and International Society for Forensic Radiology and Imaging (ISFRI) paediatric task forces were emailed the survey in May 2016, with reminder messages sent every 3 months. We included for review all responses returned by May 2017 .

\section{Survey findings}

In total, we invited 25 imaging centres to complete the survey questionnaire. We initially received responses from 23 centres (23/25, 92\%); however 3 of these centres either reported that they did not perform postmortem CT for paediatric cases, or only did so on a research basis - not as part of clinical care. Therefore, we included a total of 20 centres $(20 / 25,80 \%)$ in our final analysis, comprising 62 different paediatric postmortem CT protocols for review.

The surveys were completed by consultant radiologists $(8 / 20$, $40 \%)$, pathologists $(6 / 20,30 \%)$, both radiologists and pathologists together $(2 / 20,10 \%)$, radiographers/technologists $(2 / 20$, $10 \%)$ and both radiologists and radiographers together $(2 / 20$, $10 \%)$.

The responses originated from several countries, including:

- Europe (11/20, 55\%) - United Kingdom (3), Denmark (1), Italy (1), the Netherlands (2), Poland (3) and Switzerland (1);

- Oceania (4/20, 20\%) - Australia (3) and New Zealand (1);

- North America $(4 / 20,20 \%)$ - United States (3) and Canada (1); and

- Asia $(1 / 20,5 \%)$ - Japan (1).
Table 2 Imaging approach to paediatric postmortem CT based on survey responses from 20 centres, with consensus-recommended parameters in bold

\begin{tabular}{|c|c|}
\hline Imaging approach & $\begin{array}{l}\text { Responses } n \\
(\%)\end{array}$ \\
\hline \multicolumn{2}{|l|}{ Scanner } \\
\hline GE Healthcare & $5(25)$ \\
\hline Philips & $3(15)$ \\
\hline Siemens & $9(45)$ \\
\hline Toshiba & $2(10)$ \\
\hline Hitachi & $1(5)$ \\
\hline \multicolumn{2}{|l|}{ Scanner location } \\
\hline Hospital or clinic & $9(45)$ \\
\hline Forensic centre/mortuary & $11(55)$ \\
\hline \multicolumn{2}{|l|}{ Case types (not mutually exclusive) } \\
\hline All paediatric cases & $7(35)$ \\
\hline Infants $(<1$ year $)$ & $7(35)$ \\
\hline Stillbirths & $2(10)$ \\
\hline Fetal cases & $4(20)$ \\
\hline Other: & $10(50)$ \\
\hline Missing information on survey & $2(10)$ \\
\hline Special request by clinical team & $3(15)$ \\
\hline $\begin{array}{l}\text { Cause specific (e.g. hanging, abuse, trauma, burns related } \\
\text { injuries) }\end{array}$ & $5(25)$ \\
\hline \multicolumn{2}{|l|}{ Image interpretations } \\
\hline Radiologist & $9(45)$ \\
\hline Pathologist & $3(15)$ \\
\hline Co-reported by radiologists and pathologists & $8(40)$ \\
\hline \multicolumn{2}{|l|}{ Image acquisition } \\
\hline Radiographer/technologist & $13(65)$ \\
\hline Mortuary technician & $3(15)$ \\
\hline Pathologist & $3(15)$ \\
\hline Forensic physician (radiologist or dentist) & $1(5)$ \\
\hline \multicolumn{2}{|l|}{ Body part imaged (not mutually exclusive) } \\
\hline Whole body & $12(60)$ \\
\hline Whole body + single anatomical area & $8(40)$ \\
\hline $\begin{array}{l}\text { Single anatomical areas (can be used to make whole-body } \\
\text { scan) }\end{array}$ & $16(80)$ \\
\hline Head/neck & $14(70)$ \\
\hline Thorax, abdomen, pelvis & $8(40)$ \\
\hline Extremities & $5(25)$ \\
\hline Others (e.g., thorax, shoulders, pelvis-toes) & $4(20)$ \\
\hline \multicolumn{2}{|l|}{ Energy source } \\
\hline Single-source only & $17(85)$ \\
\hline Dual-source only & $1(5)$ \\
\hline Both single- and dual-source & $2(10)$ \\
\hline \multicolumn{2}{|l|}{ Dose modulation } \\
\hline Yes & $8(40)$ \\
\hline No & $6(30)$ \\
\hline On some protocols & $4(20)$ \\
\hline Unknown & $2(10)$ \\
\hline \multicolumn{2}{|l|}{ Detector collimator (mm) } \\
\hline $0.5-1.0$ & $19(95)$ \\
\hline 1.25 & $1(5)$ \\
\hline \multicolumn{2}{|l|}{ Image reconstructions } \\
\hline Soft tissue & $19(95)$ \\
\hline Bone & $20(100)$ \\
\hline Brain & $7(35)$ \\
\hline Lung & $9(45)$ \\
\hline $\begin{array}{l}\text { Other (mediastinum, metal reduction, vendor-specific } \\
\text { anatomical filter) }\end{array}$ & $4(20)$ \\
\hline \multicolumn{2}{|l|}{ Image reformats } \\
\hline Coronal & $17(85)$ \\
\hline Sagittal & 15 (75) \\
\hline $\begin{array}{l}\text { Maximum-intensity projection (MIP) or } \\
\text { minimum-intensity projection (MiniPs) }\end{array}$ & $6(30)$ \\
\hline Volume rendering & $11(55)$ \\
\hline Other (non-standard, special request formats) & $4(20)$ \\
\hline
\end{tabular}

The findings from the survey and pooled review of imaging protocols are described next and summarised in Tables 2 and 3 .

Most centres in this survey performed their imaging on a CT scanner dedicated to forensic examinations $(11 / 20,55 \%)$, with the remainder based in a hospital or clinic environment $(9 / 20,45 \%)$. 
Table 3 Paediatric postmortem CT protocol parameters based on the 62 separate CT protocols submitted, with consensus-recommended parameters in bold

\begin{tabular}{|c|c|}
\hline Imaging parameters & $\begin{array}{l}\text { Responses } n \\
(\%)\end{array}$ \\
\hline \multicolumn{2}{|l|}{ Kilovoltage peak $(\mathrm{kVp})$} \\
\hline 80 & $3(4.8)$ \\
\hline 100 & $10(16.1)$ \\
\hline 120 & $37(59.7)$ \\
\hline 130 & $2(3.2)$ \\
\hline Other (single $\mathrm{kV}$ not indicated) & $2(3.2)$ \\
\hline Unknown & $4(6.4)$ \\
\hline Dual source: $80 / 140-150$ & $4(6.4)$ \\
\hline \multicolumn{2}{|l|}{ Milliampere seconds $(\mathrm{mA} / \mathrm{mAs})$} \\
\hline$<100$ & $7(11.3)$ \\
\hline $100-199$ & $6(9.7)$ \\
\hline 200-299 & $12(19.4)$ \\
\hline $300-399$ & $8(12.9)$ \\
\hline $400-500$ & $9(14.5)$ \\
\hline Unknown & $4(6.4)$ \\
\hline Other (range of $\mathrm{mA} / \mathrm{mAs}$ listed) & $2(3.2)$ \\
\hline Dose modulation listed $(\mathrm{mA} / \mathrm{mAs}$ not defined) & $11(17.7)$ \\
\hline Dual source $375 / 630 \mathrm{mAs}$ & $3(4.8)$ \\
\hline \multicolumn{2}{|l|}{ Dose modulation (within protocols) } \\
\hline On & $24(38.7)$ \\
\hline Off & $31(50.0)$ \\
\hline Unknown & $7(11.3)$ \\
\hline \multicolumn{2}{|l|}{ Matrix } \\
\hline $512 \times 512$ & $26(41.9)$ \\
\hline Not reported & $36(58.1)$ \\
\hline \multicolumn{2}{|l|}{ Scan field of view (SFOV) $(\mathrm{mm})$} \\
\hline$\leq 150$ & $1(1.6)$ \\
\hline$>150-300$ & $6(9.7)$ \\
\hline$>300-450$ & $5(8.1)$ \\
\hline$>450$ (largest reported as $750 \mathrm{~mm}$ ) & $4(6.4)$ \\
\hline Adjusted to patient size & $18(29.0)$ \\
\hline Unknown & $19(30.6)$ \\
\hline Other (extended FOV) & $1(1.6)$ \\
\hline $\begin{array}{l}\text { Scanner defined (preset based on anatomical area within } \\
\text { the selected protocol) }\end{array}$ & $8(12.9)$ \\
\hline \multicolumn{2}{|l|}{ Pitch } \\
\hline & $3(4.8)$ \\
\hline$\overline{0} .5-0.8$ & $29(46.8)$ \\
\hline $0.81-0.99$ & $13(20.9)$ \\
\hline $1.0-1.2$ & $4(6.4)$ \\
\hline$>1.2$ (highest reported as 1.44 ) & $2(3.2)$ \\
\hline Unknown & $7(11.3)$ \\
\hline Other (not defined, given as range $0.35-0.80$ ) & $4(6.4)$ \\
\hline \multicolumn{2}{|l|}{ Rotation time (s) } \\
\hline$<0.5$ & $1(1.6)$ \\
\hline 0.5 & $9(14.5)$ \\
\hline$>0.5-0.99$ & $14(22.6)$ \\
\hline 1.0 & $14(22.6)$ \\
\hline Unknown & $20(32.3)$ \\
\hline $\begin{array}{l}\text { Other (rotation not defined, given as range } 0.5-1.0 \text {; dual } \\
\text { source } 0.6,0.5 \text { ) }\end{array}$ & $4(6.4)$ \\
\hline \multicolumn{2}{|l|}{ Slice thickness (mm) } \\
\hline$\leq 0.75$ & $34(54.8)$ \\
\hline $0.75-<1.0$ & $7(11.3)$ \\
\hline 1.0 & $14(22.5)$ \\
\hline$>1.0$ (largest reported as $5 \mathrm{~mm}$ ) & $3(4.8)$ \\
\hline Other (given as a range: $0.6-1.0$ or $0.6-2.0$ ) & $3(4.8)$ \\
\hline Unknown & $1(1.6)$ \\
\hline
\end{tabular}

Referral indications for postmortem CT were varied, with some centres accepting all paediatric cases referred for imaging $(7 / 20,35 \%)$ or all infants younger than 1 year $(7 / 20,35 \%)$. Just less than half of all centres imaged cases referred because of a special request from the clinical team, or for a variety of specific causes (spanning a wide range of scenarios such as hanging, abuse, trauma, burn-related injuries; 5/20, 25\%).

Image acquisition was mainly performed by the radiographer or technologist $(13 / 20,65 \%)$, although at some centres the mortuary staff $(3 / 20,15 \%)$, forensic physician $(1 / 20,5 \%)$ or pathologist $(3 / 20,15 \%)$ would operate the CT machinery. Image reporting was usually undertaken by a radiologist $(9 / 20,45 \%)$ or co-reported by a radiologist and pathologist $(8 / 20,40 \%)$.

There was the expected variation in the CT scanner vender and models used; however the most popular vendors included Siemens $(9 / 20,45 \%)$ and GE Healthcare $(5 / 20,25 \%)$. Almost all scanners were single-source scanners $(17 / 20,85 \%)$ and utilised a detector collimation of $0.5-1.0 \mathrm{~mm}(19 / 20,95 \%)$.

Body coverage was variable among centres, with most performing whole-body imaging but in different ways among and within centres. Some routinely imaged the whole body in one examination $(12 / 20,60 \%$, i.e. vertex to feet), whilst others also sometimes performed imaging of separate single-body regions (e.g., head, thorax, abdomen/pelvis) to make up a wholebody study, and this did not routinely include extremities (16/ $20,80 \%$ ). At some centres imaging of just one body region also occurred and was dependent on the referral indication (e.g., thorax for rib fractures, head/neck for traumatic brain injury).

From reviewing individual postmortem CT protocols, we noted that the following parameters were most commonly used: $120 \mathrm{kVp}$ (37/62, 59.7\%), 200-299 mAs (12/62, $19.4 \%), 0.5-0.8$ pitch $(29 / 62,46.8 \%), 1$-s rotation time (14/ $62,22.6 \%)$ and slice thickness of $\leq 0.75 \mathrm{~mm}(34 / 62,54.8 \%)$.

There were a variety of responses concerning scan field of view, with most protocols not specifically including this feature $(19 / 62,30.6 \%)$. Of those that did report the scan field of view, most protocols adjusted to patient size (attempting a scan field of view as small as possible, 18/62, 29\%). Many protocols did not state matrix size $(36 / 62,58.1 \%)$, but where this was listed it was reported as $512 \times 512(26 / 62,41.9 \%)$. The dose modulation function was stated as "off" in 50\% (31/62) of cases, with the remainder of protocols either not listing this feature $(7 / 62$, $11.3 \%)$ or having this function "on" (24/62, 38.7\%).

All centres reported that they routinely provided bone imaging algorithms for their postmortem CT studies, with the majority also performing soft-tissue algorithms (19/20, $95 \%)$. Many centres also provided dedicated brain (7/20, $35 \%)$ and lung $(9 / 20,45 \%)$ algorithms for head/neck and thoracic imaging, respectively.

In terms of multiplanar reformatting, the commonest reconstructions included coronal $(17 / 20,85 \%)$ and sagittal (15/20, $75 \%$ ) planes. Volume-rendering reformats were provided in more than half of centres $(11 / 20,55 \%)$. Some centres also produced maximum- or minimum-intensity projections $(6 / 20,30 \%)$ or non-standard oblique reformats depending on pathology and special request $(4 / 20,20 \%)$.

\section{Consensus formation}

Survey responses were presented at the postmortem imaging task force session of the annual ESPR conference in June 2017 in Davos, Switzerland. Based on the survey results, a recommended paediatric postmortem $\mathrm{CT}$ imaging protocol 
was proposed at this meeting, developed by the leading authors. The final version of this manuscript was circulated among members of ISFRI and ESPR in September 2018 for consensus approval and was formally endorsed by both the ISFRI and ESPR board prior to submission for publication.

\section{Recommended protocol}

Based on described current practices using the most commonly reported imaging parameters, a paediatric postmortem CT protocol has been devised and is shown in Table 4 . This could be easily achieved with minor modifications to most CT scanner models from all vendors at most centres.

We provide a pictorial representation of recommended whole-body scan coverage, which could be printed and posted within the $\mathrm{CT}$ control room as a reminder of how to perform a paediatric postmortem CT (Fig. 1). For ease of scanning we recommend a single scan through the whole body using the recommended imaging parameters (Table 4), with separate reconstruction algorithms for brain and body.

Whilst imaging of the extremities during paediatric postmortem CT is achievable and recommended, this is predominantly for bone assessment in the setting of trauma and might not be necessary where good-quality skeletal radiographs are available.

\section{Discussion}

The survey revealed common practices of paediatric postmortem CT imaging at several international centres with significant experience in forensic and postmortem imaging. Our paediatric postmortem CT protocol has been recommended and agreed in consensus, which allows for future standardisation of image

Table 4 Recommended paediatric postmortem CT imaging protocol

\begin{tabular}{|c|c|}
\hline \multicolumn{2}{|l|}{ Reporting and referrals } \\
\hline Case types & $\begin{array}{l}\text { Practitioner-dependent and } \\
\text { case-specific }\end{array}$ \\
\hline Image acquisition & Performed by trained radiographer \\
\hline Image interpretation & Performed by qualified radiologist \\
\hline \multicolumn{2}{|l|}{ Image acquisition } \\
\hline Coverage & Whole body (vertex to extremities) \\
\hline Kilovoltage peak $(\mathrm{kVp})$ & 120 \\
\hline Milliampere seconds (mAs) & 200-299 \\
\hline Pitch & $0.5-0.8$ \\
\hline Slice thickness (mm) & $\leq 0.75$ \\
\hline Energy source & $\overline{\text { Single source }}$ \\
\hline Dose modulation & Off \\
\hline Scan field of view (SFOV) (mm) & Adjust to patient size (small as possible) \\
\hline Detector collimator $(\mathrm{mm})$ & $0.5-1.0$ \\
\hline Rotation time $(\mathrm{s})$ & 1.0 \\
\hline \multirow{4}{*}{$\begin{array}{l}\text { Matrix } \\
\text { Kernel/filter/algorithm }\end{array}$} & $512 \times 512$ \\
\hline & Soft-tissue and bone (whole body) \\
\hline & Brain (brain coverage) \\
\hline & Lung (thoracic coverage) \\
\hline Reformats & Coronal, sagittal, volume rendering \\
\hline
\end{tabular}

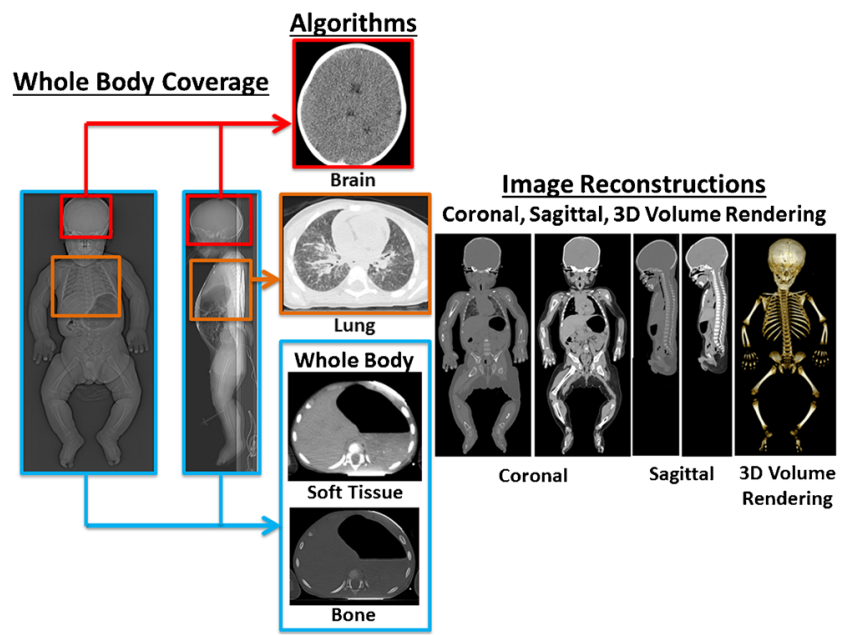

Fig. 1 Representation of the recommended joint European Society of Paediatric Radiology (ESPR) and International Society for Forensic Radiology and Imaging (ISFRI) postmortem CT imaging body coverage. The blue box represents the whole-body coverage and area for application of soft-tissue and bone algorithms. The red box represents the region for application of the brain algorithm, and the purple box the lung algorithm. Image reconstruction planes (both coronal and sagittal) are included

acquisition, improved ease of reporting - particularly when requesting external reviewer or expert opinion on cases - as well as multicentre collaborations.

Compared to several large paediatric case series utilising postmortem CT imaging dating to 2006 (Table 1), our recommended imaging parameters fall readily within those being performed worldwide. The kilovoltage of $120 \mathrm{kV}$, current of 200-299 mAs, detector collimation of $0.5-1.0 \mathrm{~cm}$ and rotation time of $1 \mathrm{~s}$ are already in common practice. It is also common practice for $\mathrm{CT}$ imaging to be reported by trained radiologists, and for the appropriate bone and soft-tissue algorithms to be used. Many published studies also reported routinely reviewing multiplanar reconstructions (i.e. coronal, sagittal planes) as well as volume-rendering reformats.

The main differences between our recommendations and the published literature include methods by which whole-body coverage is achieved, and image slice thickness. For body coverage, some papers report imaging from vertex to feet [8], others from vertex to pelvis [5]; some image the head and body separately $[3,4,10]$ or individual body areas, as required [13]. For simplicity and ease of adopting this protocol, we recommend imaging the entire body (from vertex to extremities) in a single examination, rather than imaging the head and neck in one examination and the thorax, abdomen, pelvis and lower limbs as a second separate examination. It should be noted, however, that whilst imaging the extremities is useful in situations relating to major trauma or obvious external injuries, the reduced inherent resolution of the $\mathrm{CT}$ examination compared to plain radiography can result in missing subtle fractures such as classic metaphyseal lesions. Therefore, 
if there is concern for physical abuse, one should not dismiss performing additional radiographs (particularly of the limbs). To the best of our knowledge, no studies have assessed diagnostic accuracy rates of $\mathrm{CT}$ imaging versus plain radiography in the identification of classic metaphyseal lesions, although from anecdotal experience this is well recognised and might represent an area for further evidence-based research.

We recommend a slice thickness of $\leq 0.75 \mathrm{~mm}$, whereas several reported studies in the literature used slice thicknesses ranging $1-10 \mathrm{~mm}$. This might have been a result of limitations of older scanner models, which are likely to have now been updated within most hospitals and forensic centres. Nevertheless, where centres exist with models that cannot achieve slice thicknesses below $0.75 \mathrm{~mm}$, we suggest imaging at the thinnest possible slice on the equipment available.

One factor we include in our recommended protocol that is not specifically mentioned within the published literature relates to non-usage of the dose modulation function (otherwise known as automated tube current modulation or automatic exposure control). The primary advantage of this technique is the ability to reduce the radiation dosage from a CT study without significantly influencing image quality. This is achieved by automatically adjusting the tube current with differences in soft-tissue attenuation and the body part being scanned [14]. Whilst this offers obvious advantages when imaging live children [15], these risks do not apply in the postmortem population. Adjusting and varying the tube current unnecessarily, especially using different methods of dose modulation by different $\mathrm{CT}$ vendors, might therefore introduce variability in signal to noise ratio and thus image quality [16]. In our review of paediatric postmortem CT protocols, this is already "off" in 50\% of cases and should not be used for paediatric postmortem CT imaging.

As with all studies, our retrospective self-reported study has several inherent limitations. The first relates to the relatively small number, and apparent European bias in the centres surveyed. Despite this, our results reflect the best expertise available given the high response rate from task force members from two international societies specifically established to examine this issue. Further, most published studies on paediatric postmortem CT imaging stem from Europe (Table 1).

The second limitation relates to the survey questions. These questions were designed to be concise, easy to understand and relevant to postmortem CT imaging parameters. We acknowledge that there are many other aspects surrounding the running of a paediatric postmortem CT imaging service that would have been interesting and useful to have studied. These could have included further details on imaging referral patterns and case numbers, reporting environment (e.g., standardised reporting, double reporting, presence of multi-disciplinary team meetings) and issues regarding postmortem imaging training and reporting (e.g., years of experience of technologists and reporters).
Although this would have been valuable to collect, we believe they would have made the survey more time-consuming to complete and threaten to lower our response rates from society members. Furthermore, these questions would have detracted from the main purpose of the survey, which was to produce recommendations specifically for postmortem CT imaging parameters. Many of the other issues described therefore fall outside this remit.

Finally, our recommendations are based on the current common practices of the majority of centres. To our knowledge no published CT imaging optimisation studies show a direct comparison between image quality or diagnostic accuracy with different parameters in paediatric postmortem cases. Our recommendations are therefore based on a pragmatic approach, easily adapted for a range of patient sizes and ages and easily implemented on most if not all CT scanners. The recommendations are intended to standardise paediatric postmortem CT imaging acquisition to allow for consistent image quality for diagnosis and multicentre sharing of data for future research collaboration studies. It also offers centres wishing to start paediatric postmortem $\mathrm{CT}$ as a new service a guide for setting up their local protocols.

Acknowledgements Dr. Susan C. Shelmerdine is supported by a Research Councils United Kingdom and United Kingdom Research and Innovation Fellowship, and Medical Research Council Clinical Research Training Fellowship (Grant Ref: MR/R00218/1). This award is jointly funded by the Royal College of Radiologists. Dr. Owen J. Arthurs is funded by a National Institute for Health Research Career Development Fellowship (NIHR-CDF-2017-10-037).

This work was undertaken at GOSH/ICH, UCLH/UCL who received a proportion of funding from the United Kingdom Department of Health's NIHR Biomedical Research Centre funding scheme. This article presents independent research funded by the Medical Research Council, Royal College of Radiologists and National Institute for Health Research, and the views expressed are those of the authors and not necessarily those of these institutions, the National Health Service or the Department of Health.

\section{Compliance with ethical standards}

\section{Conflicts of interest None}

Open Access This article is distributed under the terms of the Creative Commons Attribution 4.0 International License (http:// creativecommons.org/licenses/by/4.0/), which permits unrestricted use, distribution, and reproduction in any medium, provided you give appropriate credit to the original author(s) and the source, provide a link to the Creative Commons license, and indicate if changes were made.

Publisher's note Springer Nature remains neutral with regard to jurisdictional claims in published maps and institutional affiliations.

\section{References}

1. Maskell G, Wells M (2012) RCR/RCPath statement on standards for medico-legal post-mortem cross-sectional imaging in adults. Royal College of Radiologists, London

2. Rutty GN, Morgan B, Robinson C et al (2017) Diagnostic accuracy of post-mortem CT with targeted coronary angiography versus 
autopsy for coroner-requested post-mortem investigations: a prospective, masked, comparison study. Lancet 390:145-154

3. Arthurs OJ, Guy A, Thayyil S et al (2016) Comparison of diagnostic performance for perinatal and paediatric post-mortem imaging: CT versus MRI. Eur Radiol 26:2327-2336

4. Krentz BV, Alamo L, Grimm J et al (2016) Performance of postmortem CT compared to autopsy in children. Int J Legal Med 130: 1089-1099

5. Noda Y, Yoshimura K, Tsuji S et al (2013) Postmortem computed tomography imaging in the investigation of nontraumatic death in infants and children. Biomed Res Int 2013:327903

6. Shelmerdine SC, Hutchinson JC, Al-Sarraj S et al (2018) British Neuropathological Society and International Society of Forensic Radiology and Imaging expert consensus statement for post mortem neurological imaging. Neuropathol Appl Neurobiol 44:663-672

7. Sieswerda-Hoogendoorn T, Soerdjbalie-Maikoe V, de Bakker H, van Rijn RR (2014) Postmortem CT compared to autopsy in children; concordance in a forensic setting. Int J Legal Med 128:957-965

8. Proisy M, Marchand AJ, Loget P et al (2013) Whole-body post-mortem computed tomography compared with autopsy in the investigation of unexpected death in infants and children. Eur Radiol 23:1711-1719

9. Royal College of Pathologists (2016) New guidelines for the investigation of sudden unexplained death in infancy launched. https:// www.rcpath.org/discover-pathology/news/new-guidelines-for-theinvestigation-of-sudden-unexpected-death-in-infancy-launched. html. Accessed 29 Nov 2018
10. van Rijn RR, Beek EJ, van de Putte EM et al (2017) The value of postmortem computed tomography in paediatric natural cause of death: a Dutch observational study. Pediatr Radiol 47:1514-1522

11. Sonnemans LJP, Vester MEM, Kolsteren EEM et al (2018) Dutch guideline for clinical foetal-neonatal and paediatric post-mortem radiology, including a review of literature. Eur J Pediatr 177:791-803

12. Arthurs OJ, van Rijn RR, Sebire NJ (2014) Current status of paediatric post-mortem imaging: an ESPR questionnaire-based survey. Pediatr Radiol 44:244-251

13. Oyake Y, Aoki T, Shiotani S et al (2006) Postmortem computed tomography for detecting causes of sudden death in infants and children: retrospective review of cases. Radiat Med 24:493-502

14. Kalra MK, Maher MM, Toth TL et al (2004) Techniques and applications of automatic tube current modulation for CT. Radiology 233:649-657

15. Lee C, Pearce MS, Salotti JA et al (2016) Reduction in radiation doses from paediatric CT scans in Great Britain. Br J Radiol 89: 20150305

16. Papadakis AE, Perisinakis K, Damilakis J (2014) Automatic exposure control in CT: the effect of patient size, anatomical region and prescribed modulation strength on tube current and image quality. Eur Radiol 24:2520-2531

\section{Affiliations}

Susan C. Shelmerdine ${ }^{1,2}$ (D) $\cdot$ Chandra Y. Gerrard ${ }^{3} \cdot$ Padma Rao $^{4} \cdot{\text { Matthew } \text { Lynch }^{4} \text { • Jeroen Kroll }}^{5} \cdot$ Dan Martin $^{6}$. Elka Miller ${ }^{7} \cdot$ Laura Filograna $^{8} \cdot$ Rosa Maria Martinez $^{9} \cdot$ Odey Ukpo $^{10} \cdot$ Barry Daly $^{11} \cdot$ Hideki Hyodoh $^{12} \cdot$ Karl Johnson $^{13}$. Andrew Watt ${ }^{14}$. Ajay Taranath ${ }^{15}$. Scott Brown ${ }^{15}$. David Perry ${ }^{16} \cdot$ Lene Warner Thorup Boel ${ }^{17}$. Aleksandra Borowska-Solonynko ${ }^{18} \cdot$ Rick van Rijn $^{19} \cdot$ Willemijn Klein ${ }^{20} \cdot$ Elspeth Whitby ${ }^{21} \cdot$ Owen J. Arthurs ${ }^{1,2}$

1 Department of Clinical Radiology, Great Ormond Street Hospital for Children,

London, UK

2 UCL Great Ormond Street Institute of Child Health, Great Ormond Street Hospital for Children,

London, UK

3 Department of Radiology, University of New Mexico, Albuquerque, NM, USA

4 Department of Medical Imaging, Victorian Institute of Forensic Medicine \& Royal Children's Hospital,

Melbourne, Australia

5 Department of Radiology, Maastricht University Medical Center, Maastricht, the Netherlands

6 Department of Radiology, Gold Coast University Hospital, Gold Coast, Australia

7 Department of Medical Imaging, Children's Hospital of Eastern Ontario,

Ottawa, ON, Canada
8 Department of Diagnostic and Interventional Radiology, "Tor Vergata" University of Rome, Rome, Italy

9 Institute of Forensic Medicine (Virtopsy), University of Zurich, Zurich, Switzerland

10 Los Angeles County Medical Examiner-Coroner Office, Los Angeles, CA, USA

11 Office of the Chief Medical Examiner, University of Maryland, Baltimore, MD, USA

12 Center for Cause of Death Investigation, Hokkaido University Graduate School of Medicine,

Sapporo, Japan

13 Department of Radiology, Birmingham Children's Hospital, Birmingham, UK

14 Department of Diagnostic Imaging \& Clinical Physics, The Royal Hospital for Children,

Glasgow, Scotland, UK

15 Department of Medical Imaging, Women's and Children's Hospital, North Adelaide, South Australia, Australia 
16 Radiology Department, National Women's Health and Starship Children's Hospital, Auckland City Hospital,

Auckland, New Zealand

17 Department of Forensic Medicine, Aarhus University, Aarhus, Denmark

18 Department of Forensic Medicine, Medical University of Warsaw, Warsaw, Poland
19

Department of Radiology, Emma Children's Hospital, Academic Medical Center Amsterdam,

Amsterdam, the Netherlands

20 Department of Radiology and Nuclear Medicine, Radboud University Medical Center,

Nijmegen, the Netherlands

21 Academic Unit of Radiology, University of Sheffield, Sheffield, UK 